\title{
Identification of microRNAs in acute respiratory distress syndrome based on microRNA expression profile in rats
}

\author{
YINGHUI GUAN $^{1}$, XIANG JIN ${ }^{1}$, XINGANG LIU ${ }^{2}$, YUANPING HUANG ${ }^{1}$, MAN WANG $^{1}$ and XIAODAN LI ${ }^{1}$ \\ Departments of ${ }^{1}$ Respiratory Medicine and ${ }^{2}$ Intensive Care Unit, The First \\ Hospital, Jilin University, Changchun, Jilin 130021, P.R. China
}

Received March 25, 2016; Accepted March 2, 2017

DOI: $10.3892 / \mathrm{mmr} .2017 .6948$

\begin{abstract}
Acute respiratory distress syndrome (ARDS) remains a severe disease associated with an $\sim 40 \%$ mortality rate and as many as 200,000 new cases annually. MicroRNAs (miRNAs) have important roles in gene regulation and cancer development. The present study aimed to identify the potential roles of miRNAs in the pathogenesis and progression of ARDS. The miRNA expression profile of the GSE57223 dataset was downloaded from the Gene Expression Omnibus database. Following data normalization, differentially expressed miRNAs were identified using the t-test method. The miRWalk database was searched to predict target genes of the identified miRNAs and then a miRNA-miRNA network with co-regulated target genes was constructed. Additionally, Gene Ontology (GO) analysis was performed for the target genes and a miRNA-miRNA functional synergistic network (MFSN) was established. GO and pathway analyses were performed for the co-regulated target genes of significant miRNAs in MFSN. Additionally, a protein-protein-interaction network was constructed for these target genes. A total of 19 miRNAs were differentially expressed between ARDS and normal lung tissue were identified. The four downregulated rno-let-7 family members were detected to have numerous co-regulated target genes and synergistic functions. Additionally, the target genes of the four miRNAs were significantly enriched the biological processes of wounding and inflammatory response. Additionally, interleukin (IL)-6 was identified as a hub protein with a high degree. The four downregulated rno-let-7 miRNAs may be involved in the inflammatory process in the pathogenesis and progression of ARDS, via the synergistic regulation of their target genes, such as IL-6. However, additional experimental validation is required.
\end{abstract}

Correspondence to: Dr Xiaodan Li, Department of Respiratory Medicine, The First Hospital, Jilin University, 71 Xinmin Street, Changchun, Jilin 130021, P.R. China

E-mail: xiaodanLixdl@163.com

Key words: acute respiratory distress syndrome, microRNA, let-7, IL-6, inflammation

\section{Introduction}

Acute respiratory distress syndrome (ARDS) is an extreme manifestation of acute injury to the lung, characterized by extensive lung inflammation, profound hypoxemia and non-cardiogenic pulmonary edema formation $(1,2)$. In addition, ARDS is a serious complication resulting from sepsis and associated with multiple organ failure, and mortality and morbidity (3). It has been estimated that $\sim 200,000$ cases of ARDS occurred annually, with a high mortality rate of $\sim 40 \%$ in the United States (4). The outcome in ARDS is complicated as the syndrome is influenced by multiple factors, such as the nature of the precipitating factors and the extent of the subsequent multiorgan failure (5).

The pathogenesis of ARDS involves inflammatory injury to the lung endothelium and epithelium (6), which is accompanied by an influx of neutrophils into the pulmonary interstitium and bronchoalveolar space (7). The activated neutrophils may damage endothelial and epithelial cells and are considered to be a crucial factor in pathogenesis and progression of ARDS (8). Endothelial injury may result in the effusion of protein-rich fluid into alveolar airspace and the damage to alveolar epithelium may increase the entry of fluid into the alveolar lumen (2).

Previous studies have performed proteomic and genomic analyses to determine the pathogenesis of lung diseases (9-11), an increasing number of studies have focused on the role of small molecules, such as microRNAs (miRNAs) in the pathogenesis of the diseases (12-14).

miRNAs are a class of non-coding small RNAs 22 nucleotides in length. miRNAs negatively regulate gene expression through translational repression or mRNA degradation by binding to 3 '-untranslated region (3'-UTR) of the target mRNA transcripts $(14,15)$. miRNAs have been reported to be extensively involved in many biological processes and associated with various diseases $(16,17)$. A considerable number of miRNAs have been observed to participate in the regulation of physiological lung function, including miRNA (miR)-17, miR-92a and miR-127 (18-20). However, how miRNAs are involved in the pathogenesis and progression of ARDS remains to be elucidated.

The present study performed miRNA microarray analysis of the GSE57223 dataset downloaded from Gene Expression Omnibus (GEO) database, and aimed to identify the potential 
regulatory roles of miRNAs in the pathogenesis and progression of ARDS in order to provide novel insights into the regulatory mechanisms of the ARDS etiology.

\section{Materials and methods}

miRNA microarray data. The datasets of GSE57223 (2) deposited in the GEO database (https://www.ncbi.nlm.nih.gov/geo) were downloaded. The data set contains miRNA expression profiles of 12 samples from 6 control normal lung tissues and 6 ARDS lung tissue from a rat model, which has previously been described (2). The platform was GPL18654 (Rat microRNA array).

Data preprocessing. The probe numbers were converted into the corresponding gene symbols. The average probe value was calculated as the expression value of the gene when multiple probes corresponded to one gene. For probes with a missing value, the k-nearest neighbor (KNN) averaging method from the impute package in R (21) was applied to fill the vacancy, with $\mathrm{k}$ in the default parameter of 10 . Subsequently, quantile normalization was conducted using the preprocessCore package in $\mathrm{R}(22)$.

Identification of differentially expressed miRNAs. Following normalization of the data, the limma package in $\mathrm{R}$ (23) was used to identify differentially expressed miRNAs between the ARDS lung tissues and the control tissues, using the t-test method. The P-value was adjusted using the Benjamini-Hochberg method (24). Significant differentially expressed miRNAs with $\mathrm{P}<0.05$ and $\mid \log 2 \mathrm{FCl}>1$ were identified.

Construction of miRNA-miRNA network with co-regulated target genes. A gene that may be targeted by more than one miRNA was termed the co-regulated target gene of these miRNAs. The miRWalk database (25), which provides 10 different types of prediction algorithms, including DIANAmT, miRanda, miRDB, miRWalk, RNAhybrid, PICTAR4, PICTAR5, PITA, RNA22 and TargetScan, was used to construct the regulatory network of miRNAs and their target genes. The database was searched to construct the regulatory network of miRNAs and their target genes. Each method may produce a prediction result. In the present study, the target genes were predicted by at least 4 different algorithms to construct the miRNA-miRNA network with co-regulated target genes, which was visualized utilizing Cytoscape software version 3.4.0 (26).

Synergistic function analysis of miRNAs and biological function analysis of their target genes. The gene symbol of target genes was transformed into gene ID and clusterProfiler package in $\mathrm{R}$ (27) was used to perform Gene Ontology (GO) enrichment analysis, particularly for the Biological Process (BP) term. A functional synergistic miRNA-miRNA interaction pair was defined when their co-regulated target genes were enriched in at least one BP term. The threshold criteria were $\mathrm{P}<0.05$ and $\mathrm{q}<0.05$ for significant $\mathrm{BP}$ terms. The miRNA interaction pairs having common target genes were filtered out to establish the miRNA-miRNA functional synergistic network (MFSN) (15).
Table I. Predicted target genes of differentially expressed miRNAs based on the mirWalk database.

\begin{tabular}{lcl}
\hline miRNA & Target genes & Regulation \\
\hline rno-miR-126 & 28 & Down \\
rno-miR-129 & 674 & Up \\
rno-let-7a & 409 & Down \\
rno-let-7b & 409 & Down \\
rno-miR-135b & 495 & Down \\
rno-let-7c & 409 & Down \\
rno-miR-103 & 757 & Up \\
rno-let-7f & 409 & Down \\
\hline
\end{tabular}

miR, microRNA.

In the MFSN, the co-regulated target genes of the significant miRNA-miRNA interaction pairs were screened for further analysis. GO and pathway analyses were conducted using the Database for Annotation, Visualization and Integrated Discovery online software (28) and the threshold for significant GO and pathway terms were $\mathrm{P}-<0.05$. Additionally, the interacted protein-protein pairs with combined score $>0.04$ were selected to establish the protein-protein-interaction (PPI) network for the co-regulated target genes using STRING version 9.1 (29), which was visualized with Cytoscape software (26). In addition, hub proteins were screened out through the analysis of topological characteristics of the PPI network.

\section{Results}

miRNAs differentially expressed between ARDS lung tissues and normal tissues. Normalization of the microarray data was performed and the medians of miRNA expression values are presented in Fig. 1. According to the defined threshold criteria, a total of 19 significant differentially expressed miRNAs were identified between ARDS lung tissues and normal tissues, with 6 upregulated miRNAs, including rno-miR-30e, rno-mir-129, rno-miR-199a, rno-miR-34b and rno-miR-103, and 13 downregulated miRNAs, namely rno-miR-122a, rno-miR-290, rno-miR-324-5p, rno-miR-35, rno-miR-223, rno-let-7f, rno-miR-26a, rno-miR-24, rno-miR-143, rno-let-7b, rno-let-7c, rno-let-7a and rno-miR-126.

miRNA-miRNA network with co-regulated target genes. Based on the prediction of mirWalk databases and the selection criterion, the target genes of eight miRNAs were screened out. Notably, rno-miR-103 had the highest number $(n=757)$ of target genes and the 4 rno-let-7 miRNAs also had high numbers $(n=409)$ of target genes (Table I). To detect the associations of the miRNAs and their target genes, the regulatory network for the 8 miRNAs with their target genes was constructed, comprising of 3590 edges and 2152 nodes (Fig. 2). Furthermore, a miRNA-miRNA network with co-regulated target genes was established, consisting of 27 miRNA-miRNA interaction pairs. As presented in Fig. 3 and Table I, the four downregulated miRNAs from the rno-let-7 family had high numbers $(n=409)$ of target genes. 


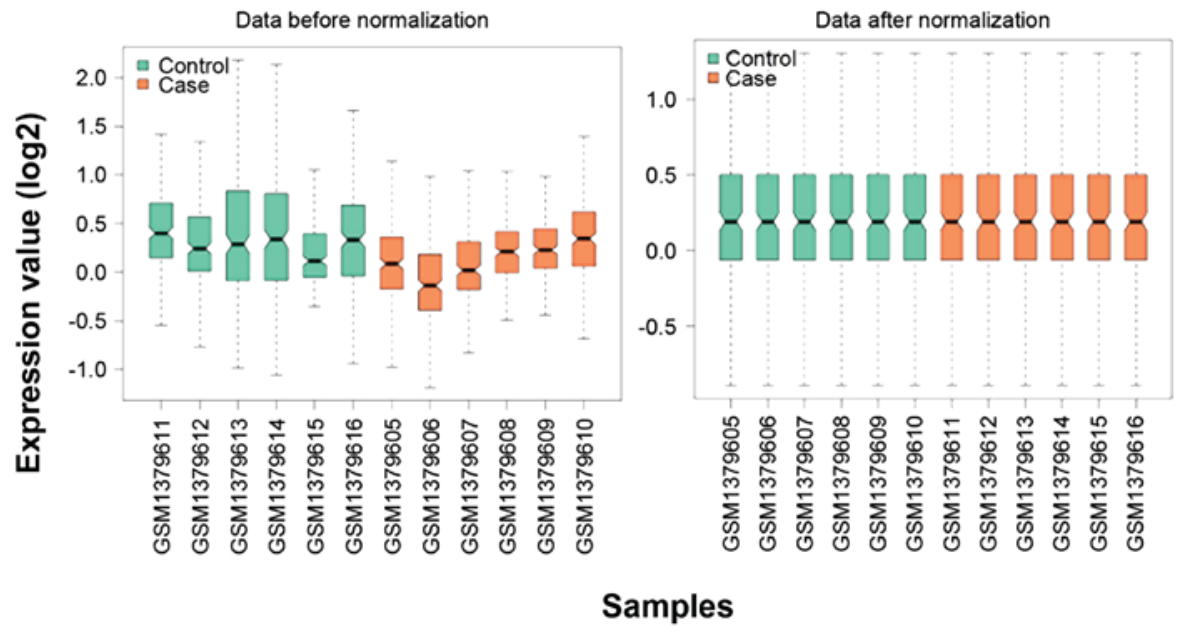

Figure 1. Normalization of the gene expression profiling. Box plots displaying the intensity log ratio distribution before and after normalization procedures. The $\mathrm{x}$-axis indicates the name of the sample in the dataset and the $\mathrm{y}$-axis indicates the value of the expression following a $\log _{2}$ transformation. Thick black lines, median.

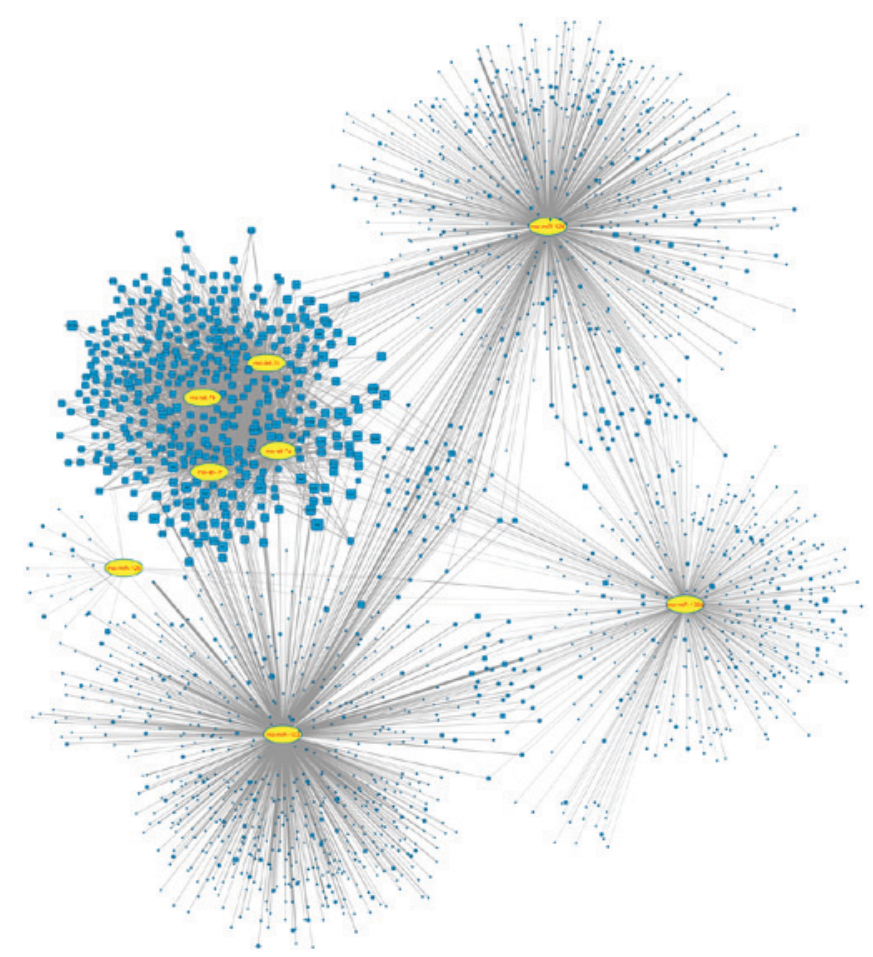

Figure 2. Regulatory network of miRNAs and their target genes. Nodes in yellow nodes represent miRNAs and nodes in blue represent target genes. miRNA, microRNA.

MFSN and biological functions of the target genes. In order to investigate the synergic functions between miRNAs, the MFSN was established, consisting of 17 miRNA-miRNA functional synergistic pairs, of the 27 identified interaction pairs with co-regulated target genes, involving a total of 7 miRNAs. As presented in Fig. 4, the 4 rno-let-7 family members were detected with synergic functions. Further functional analysis indicated that 409 target genes of the 4 rno-let- 7 family miRNAs were significantly enriched in BP terms, such as response to wounding and inflammatory response and cellular component terms, including cell fraction and insoluble fraction, and molecular function terms, such as manganese ion

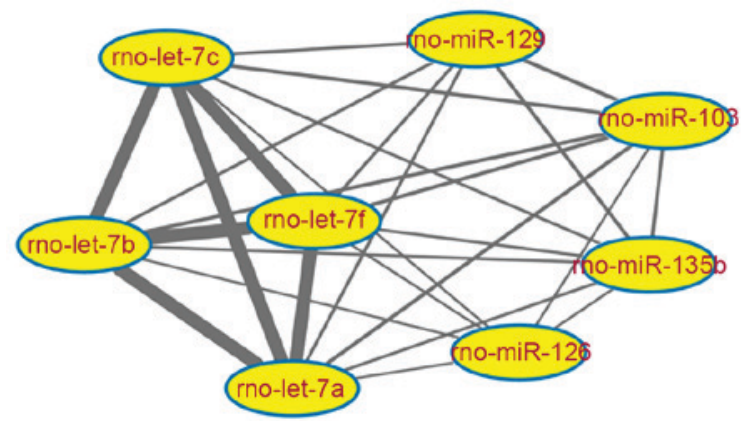

Figure 3. miRNA-miRNA network of differentially expressed miRNAs with co-regulated target genes. Yellow nodes denote differentially expressed miRNAs, and edges denote the interaction between two miRNAs. The thickness of the edge represents the number of co-regulated target genes. miRNA, microRNA.

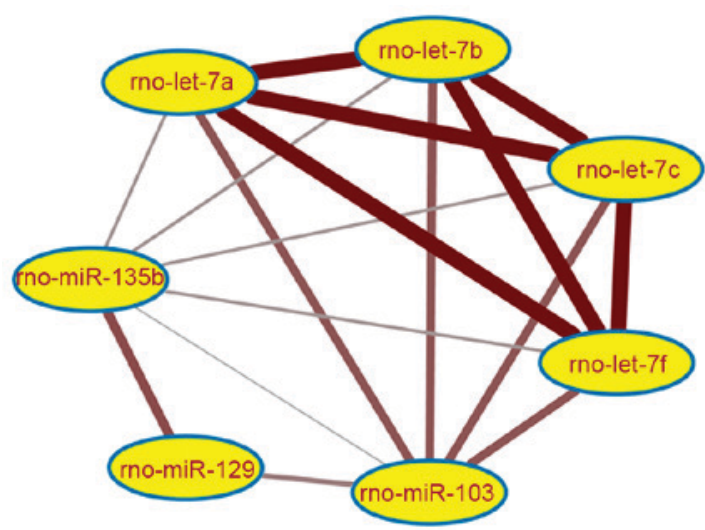

Figure 4. miRNA-miRNA functional synergistic network of differentially expressed miRNAs. Yellow nodes denote diff erentially expressed miRNAs and edges denote the synergistic relationship between two miRNAs. The thickness of the edge represents the number of biological process terms of co-regulated target genes. miRNA, microRNA.

binding and activin receptor activity. In addition, these target genes were significantly enriched in the chronic myeloid leukemia and Graft-vs.-host disease pathways (Table II). The 
Table II. Top 5 significant GO and KEGG pathway terms for the co-regulated target genes of 4 rno-let-7 microRNAs.

A, Biological processes

\begin{tabular}{llrr}
\hline Term & \multicolumn{1}{c}{ Description } & Count & P-value \\
\hline GO:0010033 & Response to organic substance & 29 & $1.66 \times 10^{-5}$ \\
GO:0009611 & Response to wounding & 17 & $1.08 \times 10^{-4}$ \\
GO:0006954 & Inflammatory response & 12 & $1.09 \times 10^{-4}$ \\
GO:0032496 & Response to lipopolysaccharide & 9 & $1.27 \times 10^{-4}$ \\
GO:0009991 & Response to extracellular stimulus & 14 & $1.36 \times 10^{-4}$ \\
\hline
\end{tabular}

$\mathrm{B}$, Cellular component

\begin{tabular}{llrr}
\hline Term & \multicolumn{1}{c}{ Description } & Count & P-value \\
\hline GO:0000267 & Cell fraction & 33 & $6.66 \times 10^{-6}$ \\
GO:0005626 & Insoluble fraction & 28 & $9.99 \times 10^{-6}$ \\
GO:0005624 & Membrane fraction & 27 & $1.14 \times 10^{-5}$ \\
GO:0031090 & Organelle membrane & 31 & $2.81 \times 10^{-5}$ \\
GO:0043235 & Receptor complex & 9 & $1.37 \times 10^{-4}$ \\
\hline
\end{tabular}

C, Molecular function

\begin{tabular}{llrr}
\hline Term & \multicolumn{1}{c}{ Description } & Count & P-value \\
\hline GO:0048037 & Cofactor binding & 13 & $4.29 \times 10^{-4}$ \\
GO:0030145 & Manganese ion binding & 7 & 0.001334536 \\
GO:0017002 & Activin receptor activity & 3 & 0.001543724 \\
GO:0050662 & Coenzyme binding & 10 & 0.002201207 \\
GO:0008289 & Lipid binding & 13 & 0.002212067 \\
\hline
\end{tabular}

D, KEGG pathway

\begin{tabular}{llrr}
\hline Term & \multicolumn{1}{c}{ Description } & Count & P-value \\
\hline rno05220 & Chronic myeloid leukemia & 6 & 0.010215393 \\
rno05332 & Graft-versus-host disease & 5 & 0.01224159 \\
rno04142 & Lysosome & 7 & 0.017086633 \\
rno00260 & Glycine, serine and threonine metabolism & 4 & 0.018428521 \\
rno04060 & Cytokine-cytokine receptor interaction & 9 & 0.022832763
\end{tabular}

GO, gene ontology; KEGG, Kyoto Encyclopedia of Genes and Genomes.

PPI network for these target genes was constructed and had 176 edges and 153 nodes (Fig. 5). Based on the topological characteristics of the PPI network, the hub proteins with high degrees were identified, including interleukin (IL)-6 (degree, 17), RNA polymerase I subunit A (degree, 11), Fas cell surface death receptor (degree, 10), IL-10 and cyclin-dependent kinase-inhibitor 1 (degree, both 9).

\section{Discussion}

ARDS is a severe syndrome of acute respiratory failure associated with multiple symptoms and influenced by numerous factors $(2,6)$. The expression of miRNAs is critically associated with cancer initiation, development and metastasis (30). The present study identified a total of 19 significant differentially expressed miRNAs between ARDS rat lung tissues and normal rat lung tissues. Notably, 4 downregulated let-7 miRNA family members (rno-let-7a, rno-let-7b, rno-let-7c and rno-let-7f) were observed to have a high number of co-regulated target genes and were recognized as miRNA-miRNA interactions with synergistic functions. Furthermore, the target genes of the miRNAs were significantly involved in the wounding and inflammatory response BP functions. In addition, IL-6 was identified as a vital hub protein based on the PPI network. 


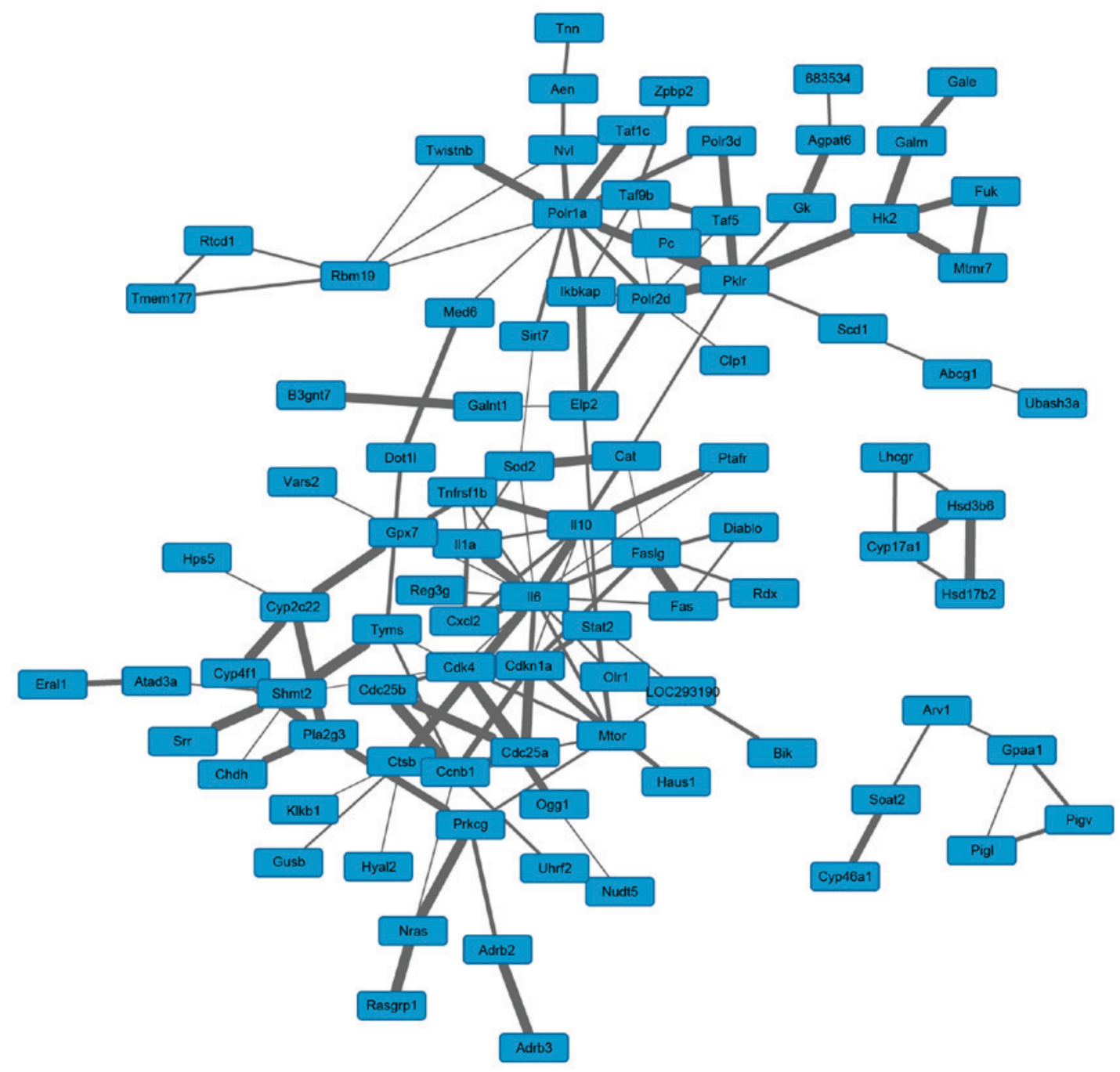

Figure 5. Protein-protein-interaction network of co-regulated target genes of four rno-let-7 microRNAs. Blue rectangles represent the target proteins and the edges represent the interaction between two proteins. The thickness of the edge represents the number of biological process terms of co-regulated target genes.

The highly conserved let-7 miRNA family members are downregulated in multiple types of cancer and the low expression of let-7 is associated with poor prognosis (31,32). By directly targeting oncogenes and inhibiting their expression levels, let-7 can act as a tumor suppressor (33). In addition, let-7 miRNA may directly limit cancer growth in the lung and the loss of its function enhances lung tumor formation $(34,35)$. In humans, let-7 miRNAs consist of 12 family members (let-7a-1, -2, -3; let-7b; let-7c; let-7d; let-7e; let-7f-1, -2; let-7g; let-7i; miR-98), which are located at 8 different chromosomal loci and the coordinate downregulation of multiple let-7 miRNAs has been identified in various tumors $(32,36)$. In the present study, the 4 rno-let-7 family members (rno-let-7a, rno-let-7b, rno-let-7c and rno-let-7f) were all downregulated in ARDS rat lung tissues and had synergistic functions, suggesting that they may synergistically suppress tumor growth associated with ARDS.

Reduced expression levels of let-7 were detected in lung cancer and were determined to be involved in the pathogenesis of this fatal disease (37). Through directly binding to the 3'-UTR of their target genes, the let-7 family members are involved in the regulation of inflammatory pathways and processes. A previous study indicated that let-7 participated in airway inflammation via the direct regulation of IL-13, a cytokine that was associated with the mediation of allergic inflammation $(38,39)$. Another cytokine, IL-6 was also reported to be targeted by let-7 and an inverse association between lower expression levels of let-7a and higher levels of IL-6 was established in various cancer types compared with normal breast, prostate, hepatocellular and lung tissues (40). A feedback loop involving nuclear factor-kB, Lin28 homolog A, let-7 and IL-6 is a critical part of the transient inflammatory signal pathway (33). IL-6 is a cytokine protein that contributes to inflammation and it has been demonstrated that elevated IL-6 expression is closely associated with increased mortality in critically ill patients with ARDS (41). IL-6 has been identified as a potential predictor for the development of ARDS in adults with severe blunt trauma (42). In addition, the high expression levels of cytokines, such as IL-6 and IL-8, were determined to be reliable markers for predicting the development and the degree of severity of the systemic inflammatory response, which may result in ARDS (43). The findings of the present study indicated that IL-6, which was significantly enriched in the inflammatory response BP, is a vital target gene of let-7 miRNAs. Therefore, let-7 miRNAs may be involved in the inflammatory process via the direct regulation of IL-6 during the progression of ARDS. 
In conclusion, the downregulated rno-let-7 miRNAs, including rno-let-7a, rno-let-7b, rno-let-7c and rno-let-7f, may have important roles in the inflammatory process during the pathogenesis and progression of ARDS through the synergistic regulation of their target genes, such as IL-6. These findings provided a novel insight into the roles of miRNAs in the mediation of ARDS development. However, further experimental experiments are required to verify the association between IL-6 and rno-let-7 miRNAs, as the current findings were based only on microarray data.

\section{References}

1. Udobi KF, Childs E and Touijer K: Acute respiratory distress syndrome. Am Fam Physician 67: 315-322, 2003.

2. Huang C, Xiao X, Chintagari NR, Breshears M, Wang Y and Liu L: MicroRNA and mRNA expression profiling in rat acute respiratory distress syndrome. BMC Med Genomics 7: 46, 2014.

3. Mikkelsen ME, Shah CV, Meyer NJ, Gaieski DF, Lyon S, Miltiades AN, Goyal M, Fuchs BD, Bellamy SL and Christie JD: The epidemiology of acute respiratory distress syndrome in patients presenting to the emergency department with severe sepsis. Shock 40: 375-381, 2013

4. Rubenfeld GD, Caldwell E, Peabody E, Weaver J, Martin DP, Neff M, Stern EJ and Hudson LD: Incidence and outcomes of acute lung injury. N Engl J Med 353: 1685-1693, 2005.

5. Wyncoll DL and Evans TW: Acute respiratory distress syndrome. Lancet 354: 497-501, 1999.

6. Matthay MA and Zemans RL: The acute respiratory distress syndrome: Pathogenesis and treatment. Annu Rev Pathol 6: 147-163, 2011.

7. Abraham E: Neutrophils and acute lung injury. Crit Care Med 31 (Suppl 4): S195-S199, 2003.

8. Grommes J and Soehnlein O: Contribution of neutrophils to acute lung injury. Mol Med 17: 293-307, 2011.

9. Magi B, Bargagli E, Bini L and Rottoli P: Proteome analysis of bronchoalveolar lavage in lung diseases. Proteomics 6 : 6354-6369, 2006.

10. Amos CI, Wu X, Broderick P, Gorlov IP, Gu J, Eisen T, Dong Q, Zhang Q, Gu X, Vijayakrishnan J, et al: Genome-wide association scan of tag SNPs identifies a susceptibility locus for lung cancer at 15q25. 1. Nat Genetics 40: 616-622, 2008.

11. Potti A, Mukherjee S, Petersen R, Dressman HK, Bild A, Koontz J, Kratzke R, Watson MA, Kelley M, Ginsburg GS, et al: A genomic strategy to refine prognosis in early-stage non-small-cell lung cancer. N Engl J Med 355: 570-580, 2006.

12. Nana-Sinkam SP, Hunter MG, Nuovo GJ, Schmittgen TD, Gelinas R, Galas D and Marsh CB: Integrating the MicroRNome into the study of lung disease. Am J Respir Crit Care Med 179: 4-10, 2009

13. Yanaihara N, Caplen N, Bowman E, Seike M, Kumamoto K, Yi M, Stephens RM, Okamoto A, Yokota J, Tanaka T, et al: Unique microRNA molecular profiles in lung cancer diagnosis and prognosis. Cancer Cell 9: 189-198, 2006.

14. Angulo M, Lecuona E and Sznajder JI: Role of MicroRNAs in lung disease. Arch Bronconeumol 48: 325-330, 2012 (In English, Spanish).

15. Xu J, Li CX, Li YS, Lv JY, Ma Y, Shao TT, Xu LD, Wang YY, Du L, Zhang YP, et al: MiRNA-miRNA synergistic network: Construction via co-regulating functional modules and disease miRNA topological features. Nucleic Acids Res 39: 825-836, 2011.

16. Ha TY: MicroRNAs in human diseases: From lung, liver and kidney diseases to infectious disease, sickle cell disease and endometrium disease. Immune Netw 11: 309-323, 2011.

17. Small EM and Olson EN: Pervasive roles of microRNAs in cardiovascular biology. Nature 469: 336-342, 2011.

18. Carraro G, El-Hashash A, Guidolin D, Tiozzo C, Turcatel G, Young BM, De Langhe SP, Bellusci S, Shi W, Parnigotto PP and Warburton D: miR-17 family of microRNAs controls FGF10-mediated embryonic lung epithelial branching morphogenesis through MAPK14 and STAT3 regulation of E-Cadherin distribution. Dev Biol 333: 238-250, 2009.

19. Lin HY, Chiang CH and Hung WC: STAT3 upregulates miR-92a to inhibit RECK expression and to promote invasiveness of lung cancer cells. Br J Cancer 109: 731-738, 2013.
20. Bhaskaran M, Wang Y, Zhang H, Weng T, Baviskar P, Guo Y, Gou D and Liu L: MicroRNA-127 modulates fetal lung development. Physiol Genomics 37: 268-278, 2009.

21. Hastie T, Tibshirani R, Narasimhan B and Chu G: Impute: Imputation for microarray data. $\mathrm{R}$ package version 1.44.0, 2001.

22. Bolstad B: PreprocessCore: A collection of pre-processing functions. R package version 1, 2013.

23. Smyth GK: Limma: Linear Models for Microarray Data. In: Bioinformatics and Computational Biology Solutions Using R and Bioconductor. Springer, New York, NY, pp397-420, 2005.

24. Hochberg Y and Benjamini Y: More powerful procedures for multiple significance testing. Stat Med 9: 811-818, 1990.

25. Dweep H, Sticht C, Pandey P and Gretz N: miRWalk-database: Prediction of possible miRNA binding sites by 'walking' the genes of three genomes. J Biomed Inform 44: 839-847, 2011.

26. Shannon P, Markiel A, Ozier O, Baliga NS, Wang JT, Ramage D, Amin N, Schwikowski B and Ideker T: Cytoscape: A software environment for integrated models of biomolecular interaction networks. Genome Res 13: 2498-2504, 2003.

27. Yu G, Wang LG, Han Y and He QY: clusterProfiler: An R package for comparing biological themes among gene clusters. OMICS 16: 284-287, 2012.

28. Dennis G Jr, Sherman BT, Hosack DA, Yang J, Gao W, Lane HC and Lempicki RA: DAVID: Database for annotation, visualization, and integrated discovery. Genome Biol 4: P3, 2003.

29. Franceschini A, Szklarczyk D, Frankild S, Kuhn M, Simonovic M, Roth A, Lin J, Minguez P, Bork P, von Mering C and Jensen LJ: STRING v9. 1: Protein-protein interaction networks, with increased coverage and integration. Nucleic Acids Res 41: D808-D815, 2013.

30. Di Leva G and Croce CM: Roles of small RNAs in tumor formation. Trends Mol Med 16: 257-267, 2010.

31. Roush S and Slack FJ: The let-7 family of microRNAs. Trends Cell Biol 18: 505-516, 2008.

32. Piskounova E, Polytarchou C, Thornton JE, LaPierre RJ, Pothoulakis C, Hagan JP, Iliopoulos D and Gregory RI: Lin28A and Lin28B inhibit let-7 microRNA biogenesis by distinct mechanisms. Cell 147: 1066-1079, 2011.

33. Iliopoulos D, Jaeger SA, Hirsch HA, Bulyk ML and Struhl K: STAT3 activation of miR-21 and miR-181b-1 via PTEN and CYLD are part of the epigenetic switch linking inflammation to cancer. Mol Cell 39: 493-506, 2010.

34. Trang P, Medina PP, Wiggins JF, Ruffino L, Kelnar K, Omotola M, Homer R, Brown D, Bader AG, Weidhaas JB and Slack FJ: Regression of murine lung tumors by the let-7 microRNA. Oncogene 29: 1580-1587, 2010

35. Esquela-Kerscher A, Trang P, Wiggins JF, Patrawala L, Cheng A, Ford L, Weidhaas JB, Brown D, Bader AG and Slack FJ: The let-7 microRNA reduces tumor growth in mouse models of lung cancer. Cell Cycle 7: 759-764, 2008

36. Shell S, Park SM, Radjabi AR, Schickel R, Kistner EO, Jewell DA, Feig C, Lengyel E and Peter ME: Let-7 expression defines two differentiation stages of cancer. Proc Natl Acad Sci USA 104: 11400-11405, 2007.

37. Takamizawa J, Konishi H, Yanagisawa K, Tomida S, Osada H, Endoh H, Harano T, Yatabe Y, Nagino M, Mitsudomi T and Takahashi T: Reduced expression of the let-7 microRNAs in human lung cancers in association with shortened postoperative survival. Cancer Res 64: 3753-3756, 2004.

38. Zhu Z, Zheng T, Homer RJ, Kim YK, Chen NY, Cohn L, Hamid Q and Elias JA: Acidic mammalian chitinase in asthmatic Th2 inflammation and IL-13 pathway activation. Science 304: 1678-1682, 2004.

39. Kumar M, Ahmad T, Sharma A, Mabalirajan U, Kulshreshtha A, Agrawal A and Ghosh B: Let-7 microRNA-mediated regulation of IL-13 and allergic airway inflammation. J Allergy Clin Immunol 128: 1077-1085. e1-e10, 2011.

40. Iliopoulos D, Hirsch HA and Struhl K: An epigenetic switch involving NF-kappaB, Lin28, Let-7 MicroRNA, and IL6 links inflammation to cell transformation. Cell 139: 693-706, 2009.

41. Marshall RP, Webb S, Hill MR, Humphries SE and Laurent GJ: Genetic polymorphisms associated with susceptibility and outcome in ARDS. Chest 121 (3 Suppl): 68S-69S, 2002.

42. Kasotakis G, Kromer M, Narsule C, Sideris A, Klein E, Tompkins R, Velmahos G and Burke P: 349: Interleukin-6 predicts acute respiratory distress syndrome in adults with severe blunt trauma. Crit Care Med 41: A82, 2013.

43. Volpin G, Cohen M, Assaf M, Meir T, Katz R and Pollack S: Cytokine levels (IL-4, IL-6, IL-8 and TGF $\beta$ ) as potential biomarkers of systemic inflammatory response in trauma patients. Int Orthop 38: 1303-1309, 2014. 